\title{
A BAND SELECTION METHOD FOR SUB-PIXEL TARGET DETECTION IN HYPERSPECTRAL IMAGES BASED ON LABORATORY AND FIELD REFLECTANCE SPECTRAL COMPARISON
}

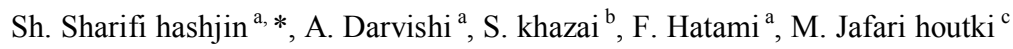 \\ ${ }^{a}$ Dept. Of Remote Sensing and GIS, Geography Faculty, University of Tehran, Iran - sh.sharifi@ut.ac.ir \\ ${ }^{\mathrm{b}}$ Dept. of Civil Engineering, Engineering Faculty, University of Imam Hussein, Tehran, Iran - skhazai@ihu.ac.ir \\ ${ }^{\mathrm{c}}$ Dept. of Geology, Science Faculty, Shahid Bahonar University of Kerman, Kerman, Iran- Avandplast@gmail.com
}

KEY WORDS: Hyperspectral, Target detection, Band selection, Target spectral, Sub-pixel

\begin{abstract}
:
In recent years, developing target detection algorithms has received growing interest in hyperspectral images. In comparison to the classification field, few studies have been done on dimension reduction or band selection for target detection in hyperspectral images. This study presents a simple method to remove bad bands from the images in a supervised manner for sub-pixel target detection. The proposed method is based on comparing field and laboratory spectra of the target of interest for detecting bad bands. For evaluation, the target detection blind test dataset is used in this study. Experimental results show that the proposed method can improve efficiency of the two well-known target detection methods, ACE and CEM.
\end{abstract}

\section{INTRODUCTION}

\subsection{General Instructions}

The hyperspectral imaging sensors with many narrow and contagious bands from the visible to the infrared region of the electromagnetic spectrum have substantially contributed for obtaining information from the objects and phenomena. In recent decades, target detection (TD) has received considerable interest in hyperspectral image processing [Geng, et al. 2014]. Nonetheless, detection of sub-pixel targets which only occupy some part of a pixel is one of the most important challenges for hyperspectral imaging applications because the signatures of such targets which are strongly mixed with their neighboring objects have a slightly deviate from the background signatures [Khazai, et al. 2013].

Although the abundant bands play an important role in the TD, the high correlation among bands makes hyperspectral data greatly redundant, leading to a problem such as 'Hughes phenomena [Hughes, 1968]. Therefore, band selection (BS), which can be used to address this problem, has received increasing attention. However, most of the existing BS methods for hyperspectral data are classification orientated and few of them are proposed specially for TD. This study presents a novel but simple TD method aimed at quickly detecting the good bands in hyperspectral images.

\section{MATERIAL}

\subsection{Target Detection}

Supervised TD problem can be considered as a one-class classification problem between the target and non-target pixels using training data from the target class only. However, a spectral signature of the target sample obtained using field or laboratory measurements [Yang, et al. 2014, Yang, et al. 2011,
Kuo. Et al. 2014, Jin, et al. 2009] is the only training data available for TD.

Target detection methods can be divided into statistical and non-statistical methods, such as clustering based methods and nearest neighboring. The statistical-based methods can also be divided into parametric and non-parametric methods.

The well-known TD methods are Constrained Energy Minimization (CEM)[Harsanyi. 1993, Chang, et al. 2000], Adaptive Cosine Estimator (ACE)[Manolakis, et al. 2003], Spectral Angle Mapper(SAM)[Kruse, et. al. 1993], Matched Filter [Manolakis, et al. 2000., Chen. and Reed, 1987], Orthogonal Subspace Projection (OSP) [Harsanyi. and Chang, 1994., Chang, 2005] and the kernel-sparse version of them [Duand, and Zhang. 2014., Zhang, Y et al. 2014., Zhang, L et al. 2014].

In comparison to the classification field, few studies have been done on dimension reduction or band selection for target detection in hyperspectral images. The purpose of this study is to present a simple but efficient band selection method for TD. In this study, two common and mostly used TD algorithms, called ACE and CEM, are used for assessment of the proposed method.

\subsection{ACE algorithm}

ACE is one of the unstructured background models that assume background statistical parameters are unknown. In this method image background can be modelled with a normal multivariate distribution as $\mathrm{B} \sim \mathrm{N}(\mu, \Gamma)$, in which $\mu$ and $\Gamma$ are mean and variance of background pixels respectively.

Considering $\mathrm{T}$ and $\mathrm{B}$ as target and background endmember with their abundance as $\alpha$ and background additive noise $\mathrm{W}$, presence and absent hypothesis of target can be modeled as the following equation:

\footnotetext{
* Corresponding author
} 


$$
\begin{aligned}
& H_{0}: \alpha_{b} \cdot B+W \\
& H_{1}: \alpha_{t} \cdot T+\alpha_{b} \cdot B+W
\end{aligned}
$$

So in this case used by Generalized Likelihood Ratio Test ACE detector defined as equation 2 [Jin, et al. 2009].

$$
D_{A C E}(X)=\frac{\left(X^{T} \Gamma^{-1} T\right)\left(T^{T} \Gamma^{-1} X\right)}{\left(X^{T} \Gamma^{-1} T\right)\left(S^{T} \Gamma^{-1} T\right)}
$$

\subsection{CEM algorithm}

In Constrained energy minimization method based on target vector $\mathrm{T}$, a filter such as $\mathrm{W}$ will be created in which after apply in hyperspectral data, the new image with highlighted pixels similar to target vector will be achieved. Using by following equation final value of each pixel in new image $(\mathrm{Y})$ will be calculated [Jin, et al. 2009].

$$
\begin{aligned}
& Y=w . r \\
& D_{C E M}(X)=\frac{T^{T} R^{-1} X}{T^{T} R^{-1} T}
\end{aligned}
$$

Where $\mathrm{T}$ is the target spectrum, $\mathrm{X}$ is the pixel spectrum, and $\mathrm{R}$ is the background correlation or covariance matrix. In CEM using a specific constraint, output energy of the desired target will be maximized respect to other pixels.

Similar to ACE, CEM method only used similarity comparison between target spectrum and image pixels and don't need to know all image endmembers.

\subsection{Data set used}

Target Detection Blind Test (TDBT) [Kerekes, J. P.] dataset, which is a real hyperspectral data set, is used for our experiments in this study. This data set includes two HyMap radiance and reflectance images of Cooke City in Montana, USA (see Fig.1). The images were collected by an airborne HyMap sensor, which has 126 spectral bands. The ground resolution of imagery data is approximately $3 \mathrm{~m}$.

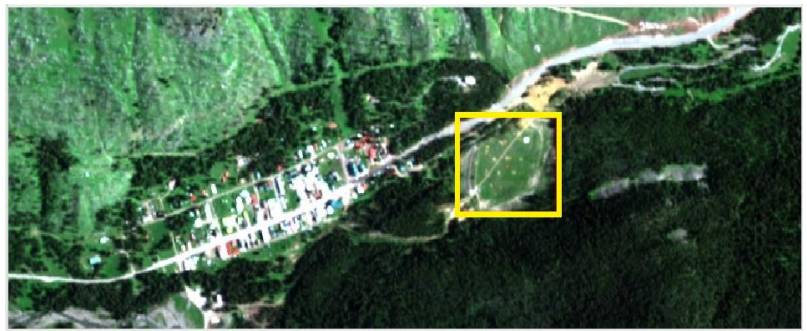

Figure 1. The true colour composite of the HyMap image. The yellow region represents location of the targets

In the image scenes, 10 sub-pixel targets were located in an open grass region (fig. 1) during the image acquisition. Table I briefly describes the characteristics of each target. In the data set, the lab and field-based reflectance spectra have 126 and 2150 bands, respectively.

\begin{tabular}{|ccc|ccl|}
\hline \multicolumn{3}{|c|}{ self-test targets } & \multicolumn{3}{c|}{ blind-test targets } \\
\hline Name & Size & Type & Name & Size & Type \\
\hline F3a & $2 \times 2 \mathrm{~m}$ & Blue Cotton & F5b & $1 \times 1 \mathrm{~m}$ & Maroon Nylon \\
F3b & $1 \times 1 \mathrm{~m}$ & Blue Cotton & F6a & $2 \times 2 \mathrm{~m}$ & Gray Nylon \\
F4a & $2 \times 2 \mathrm{~m}$ & Red Nylon & F6b & $1 \times 1 \mathrm{~m}$ & Gray Nylon \\
F4b & $1 \times 1 \mathrm{~m}$ & Red Nylon & F7a & $2 \times 2 \mathrm{~m}$ & Green Cotton \\
F5a & $2 \times 2 \mathrm{~m}$ & Maroon Nylon & F7b & $1 \times 1 \mathrm{~m}$ & Green Cotton \\
\hline
\end{tabular}

Table 1. The characteristics of the sub-pixel targets

\section{THE PROPOSED METHOD}

The idea behind our innovation in band selection is comparing the laboratory and field based reflectance spectra in order to detect bad (noisy and damaged) bands. Since the laboratory spectrum of target is measured under standard conditions with the minimized level of noise and atmospheric effects, it can be considered the ideal spectrum. On the other hand, the recorded field-based reflectance spectrum is affected by surrounding objects such as vegetation and atmospheric affects specially water vapor absorption. Obviously, the spectrum becomes progressively noisier at longer wavelengths due to normal reduction in radiance of the illumination source, the sun. However, the anomalies, bad bands, can be observed in the field based spectrum when comparing with the laboratory spectrum of the target of interest. The assumption of the proposed BS method is that the lab-field reflectance differences in the spectral bands are follows the normal distribution. Based on this assumption, the bad bands may be easily detected. Considering that most of the lab-field difference values are normal, in this study the bad bands are defined as the bands that have the difference values more than a 2.5 standard deviation away from the mean of the obtained difference values of all bands, i.e., at the significant level of 95\%. After removing the bad bands, in the second step the mean and the standard deviation of the remaining (good) bands are calculated and again bad bands are detected and removed. This process will be continued as long as the mean and the standard deviation of the difference values are smaller than a cutoff threshold.

\section{EXPERIMENTAL RESULTS}

To perform the comparison of the spectra, 2150 bands of the field spectrum are linearly resampled to 126 bands. Figure 2 shows the lab and the resampled field spectra of the sub-pixel target F3. As can be observed, using a comparison of the lab and field spectra the bad bands can be detected.

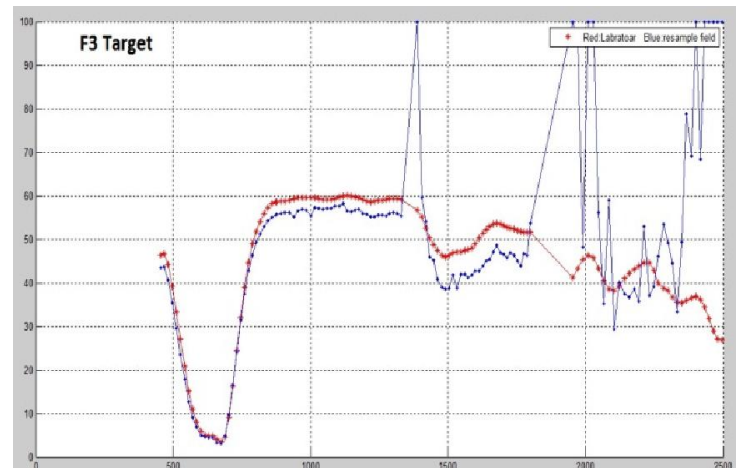

Figure 2. Laboratory (red) and resample field (blue) spectra of the subpixel target F3 
In this study, the impact of BS on the sub-pixel TD detection is investigated. Moreover, the performance of the proposed method is compared the 90 good bands provided by Gergs [Gerg, I.]. For the evaluation, two well-known sub-pixel target detection algorithms, the CEM [Harsanyi, 1993] and the ACE [Manolakis, et al. 2003], are used. Table 2 shows the number of false alarms obtained for detecting the sub-pixel targets for each combination of the TD algorithm and the BS method. From this table, the use of BS method has great impact on reducing the false alarms. Moreover, the results indicate that the proposed method significantly improves the detection performance compared to that of the Gergs method, especially for detecting the sub-pixel targets F3b, F5b, and F7b.

Easy use and computational burden reduction are advantages of this method. Moreover water absorption bands that usually manually removed in preprocessing steps of hyperspectral image processing, were eliminated automatically which indicate performance of this procedure.

Table 2. Number of false alarms obtained for detecting the targets

\begin{tabular}{|c|c|c|c|c|c|c|c|c|c|c|c|}
\hline $\begin{array}{l}\text { NO bands/BS } \\
\text { method }\end{array}$ & $\begin{array}{l}\text { TD } \\
\text { method }\end{array}$ & F7b & $\mathrm{F} 7 \mathrm{a}$ & F6b & F6a & $\mathrm{F} 5 \mathrm{~b}$ & F5a & $\mathrm{F} 4 \mathrm{~b}$ & $\mathrm{~F} 4 \mathrm{a}$ & $\mathrm{F} 3 \mathrm{~b}$ & $\mathrm{~F} 3 \mathrm{a}$ \\
\hline \multirow{2}{*}{$\begin{array}{l}\text { All } \\
\text { bands(126) }\end{array}$} & $\mathrm{ACE}$ & 61925 & 13712 & 9 & 1 & 775 & 1 & 21 & 2 & 172602 & 127271 \\
\hline & CEM & 26686 & 2876 & 102 & 28 & 348 & 19 & 316 & 39 & 142241 & 37669 \\
\hline \multirow{2}{*}{$\begin{array}{l}\text { Gergs } \\
\text { bands }(90)\end{array}$} & $\mathrm{ACE}$ & 40025 & 146679 & 3019 & 1 & 25607 & 26 & 31 & 20 & 219044 & 50849 \\
\hline & CEM & 14370 & 172119 & 1288 & 21 & 14989 & 299 & 386 & 318 & 106859 & 7240 \\
\hline \multirow{2}{*}{$\begin{array}{l}\text { Proposed } \\
\text { method }\end{array}$} & $\mathrm{ACE}$ & 2677 & 62787 & 9 & 1 & 29 & 1 & 81 & 1 & 23 & 1 \\
\hline & CEM & 2116 & 19044 & 72 & 14 & 181 & 16 & 563 & 50 & 470 & 64 \\
\hline
\end{tabular}

\section{CONCLUSION}

In this study a simple method was proposed for band selection for supervised TD. The proposed method was based on comparing field and laboratory spectra of the target of interest for detecting bad bands in hyperspectral images.

Experimental results obtained on the target detection blind test dataset showed that compared to using all bands and the bands selected by Gerges method, the proposed band selection method applied to the ACE and CEM sub-pixel TD algorithms results in improved and detection performance with decreased false alarms.

\section{REFERENCES}

[1] Geng, X., L. Ji, K. Sun, and Y. Zhao., 2014. “CEM: More Bands, Better Performance." IEEE Geoscience and Remote Sensing Letters 11(11): 1876-1880. doi:10.1109/LGRS. 2312319.

[2] Khazai, S., Safari, A., Mojaradi, B., Homayouni, S., 2013. "An Approach for Subpixel Anomaly Detection in Hyperspectral Images", IEEE Journal of Selected Topics in Applied Earth Observations and Remote Sensing. vol.6, no.2, part3, pp.769-778.

[3] Hughes, G. F., 1968. "On the mean accuracy of statistical pattern recognizers," IEEE Transactions on Information Theory, vol. IT-14, No. 1, pp. 55-63.

[4] Kerekes, J. P. and Snyder, D., "Target Detection Blind Test,” [Online]. Available: http://dirsapps.cis.rit.edu/blindtest/

[5]. Gerg, I., "MATLAB Hyperspectral Toolbox", [Online]. Available:

https://github.com/isaacgerg/matlabHyperspectralToolbox.

[6] Harsanyi, J. C., 1993. "Detection and classification of subpixel spectral signatures in hyper spectral image sequences," Ph. D. dissertation, Dept. Elect. Eng., Univ. Maryland, Baltimore, MD, USA.
[7] Manolakis, D., Marden, D. and Shaw, G. A., 2003. "Hyperspectral image processing for automatic target detection applications," LincolnLab.J., vol. 14, no. 1,pp. 79-116.

[8] Harsanyi, J. C., "Detection and classification of sub pixel spectral sign a tures in hyperspectral image sequences," Ph.D. dissertation, Dept. Elect. Eng., Univ. Maryland, Baltimore, MD, USA, (1993).

[9] Chang, C.-I., Liu, J.-M., Chieu, B.-C., Wang, C.-M., Lo, C. S., Chung, P.-C., Ren, H., Yang, C.-W. and Ma, D.-J.,

"A generalized constrained energy minimization approach to subpixel target detection for multispectral imagery,"

Optical Engineering 39(5), 1275-1281 (2000).

[10] Manolakis, D., Marden. D. and Shaw. G. A., "Hyperspectral image processing for automatic target detection applications," Lincoln Lab.J., vol. 14, no.1, pp.79-116, (2003).

[11] Kruse, F. A., Lefkoff. A. B., Boardman. J. B., Heidebrecht. K. B., Shapiro. A. T., Barloon. P. J. and Goetz. A. F. H., "The Spectral Image Processing System (SIPS) Interactive visualization and analysis of imaging spectrometer data," Remote Sens. Environ., vol.44, no.2/3, pp.145-163 , May/Jun. (1993).

[12] Manolakis, D., Shaw, G. and Keshava, N., "Comparative analysis of hyperspectral adaptive matched filter detector," in Proc. SPIE, pp. 2-17. (2000).

[13] Chen, J. Y. and Reed, S. I., "A detection algorithm for optical targets in clutter," IEEE Trans. Aerosp. Electron. Syst., vol. AES-23, no.1, pp.46-59, Jan. (1987).

[14] Harsanyi, J. C. and Chang, C.-I., "Hyperspectral image classification and dimensionality reduction: An orthogonal subspace projection approach," IEEE Trans. Geosci. Remote Sensing, vol. 32, no. 4, pp. 779-785, July (1994).

[15] Chang, C-I., "Orthogonal subspace projection (OSP) revisited: A comprehensive study and analysis," IEEE Transactions on Geoscience and Remote Sensing, vol. 43, no. 3, pp. 502-518, (2005). 
[16] Duand, B. and Zhang, L., "Target detection based on a dynamic subspace," Pattern Recog., vol. 47, no.1, pp. 344-358, Jan. (2014).

[17] Zhang, Y., Du, B. and Zhang, L., "Regularizati on frame work for target Detection in hyperspectral imagery," IEEE Geosci. Remote Sens. Lett., vol.11, no.1, pp.313-317, Jan. (2014).

[18] Zhang, L., Tao, D., Huang, X., "Sparse Transfer Manifold Embedding for Hyperspectral Target Detection". IEEE transactionson geoscience and remote sensing, vol. 52, no.2. (2014).

[19] Yang, S. and Zhenwei, S., "Sparse CEM and Sparse ACE for Hyperspectral Image Target Detection," in Geoscience and Remote Sensing Letters, IEEE, vol.11, no.12, pp.2135-2139, Dec. (2014).

[20] Yang, H., Du, Q., Su, H. and Sheng. Y., "An efficient method for supervised hyperspectral band selection," IEEE Geosci. Remote Sens. Lett., vol.8, no.1, pp.138-142, Jan. (2011).

[21] Kuo, B., Ho, H., Li, C., Hung, C. and Taur, J., "A kernel based feature selection method for SVM with RBF kernel for hyperspectral image classification," IEEE J. Sel. Topics Appl. Earth Observ. RemoteSens., vol.7, no.1,pp.317-3226, Jan. (2014).

[22] Jin, X., Paswaters, S. and Cline, H., "A comparative study of target detection algorithms for hyperspectral imagery. in Proceedings of SPIE, vol.7334, p.73341W. (2009). 